\title{
El papel de la formación inicial y permanente del profesorado para forjar lectores
}

The role of the initial and permanent training of teachers to build readers

\author{
Dra. Ester Trigo Ibáñez \\ Departamento de Didáctica \\ de la Lengua y la Literatura \\ Universidad de Cádiz \\ ester.trigo@uca.es
}

\begin{abstract}
Resumen
Puesto que la lectura es una preocupación muy extendida en la sociedad y que en el profesorado recae una gran responsabilidad a la hora de forjar lectores competentes, es vital que este colectivo cuente con una adecuada formación, tanto en su fase inicial como en la permanente. A lo largo de estas líneas hemos trazado un camino similar al que podría realizar un docente, desde la formación inicial en el Grado de Magisterio analizando el itinerario formativo de la Universidad de Cádiz - hasta las posibilidades de formación permanente del profesorado, prestando atención a las actividades ofertadas por el Centro del profesorado de Cádiz y a las propuestas de innovación de la Consejería de Educación de la Junta de Andalucía.
\end{abstract}

\begin{abstract}
Since reading is a widespread concern in society. Being aware that teachers are highly responsible of training young readers, it is vitally important for this professional group to obtain an appropriate initial and permanent training. In this paper we have drawn a similar course of which a teacher could take, from the initial training studying the Degree of Education - analysing the formative itinerary at University of Cádiz - to the possibilities of permanent training for teachers. For this, we have focused our attention on the activities offered by the teachers' centre of Cádiz and on the proposals for innovation of the Andalusian Department of Education.
\end{abstract}

Palabras clave: Formación del lector, Formación inicial, Formación permanente.

Keywords: Readers' formation, Initial training, Permanent training

\section{No te rindas, por favor no cedas [Mario Benedetti "No te rindas"] (o introducción)}

La lectura - o la falta de interés por esta actividad - es una preocupación muy extendida en las sociedades del siglo XXI. Las familias se preguntan qué pueden hacer para ayudar a sus hijos e hijas a leer; las instituciones - Ministerios, Consejerías, Ayuntamientos, bibliotecas...- - tratan de crear campañas que acerquen la lectura a la población infantil y juvenil; y las escuelas intentan hacer que sus estudiantes logren el éxito escolar con una adecuada competencia lectora y obtengan placer con la lectura tratando de navegar hacia una educación literaria. Esta preocupación social ha sido 
ampliamente estudiada en numerosas investigaciones de uno y otro lado del Atlántico (Marchamalo, 2004; Willighan, 2016).

Atendiendo al tiempo de lectura empleado en la adolescencia, Willighan (2016, p. 2) analiza los "deseos frente a la realidad en relación con el tiempo libre de los adolescentes estadounidenses". Para los padres, la lectura debería ser la principal actividad, seguida de la dedicación a las amistades; la práctica de deportes; relajarse, pensar; ver la televisión y jugar a videojuegos. Sin embargo, los cuestionarios realizados a los jóvenes dejaron ver que su actividad preferida era ver la televisión, mientras que la lectura ocupaba el último lugar. Marchamalo (2004) alude a un estudio efectuado para la Federación de Gremios de Editores y el Ministerio de Cultura en 2002 entre personas residentes en España mayores de 14 años en el que se expone que el $47 \%$ de los encuestados confiesa no haber leído nunca.

Desde nuestro trabajo como asesora de formación del ámbito lingüístico en el Centro de Profesorado de Cádiz (en adelante, CEP de Cádiz) y como profesora asociada a la Universidad de Cádiz (en adelante, UCA) en el Departamento de Didáctica de la Lengua y la Literatura, consideramos que es sumamente importante prestar atención a este aspecto y analizar cuáles son las posibilidades que el docente actual -en formación o en ejercicio - tiene para contribuir a solucionar este problema de tan alto calado social pues, como se determina en una de las conclusiones del informe de la consultora Mckinsey (2008), el nivel educativo de un país depende de la formación, motivación y aprendizaje permanente de su profesorado.

Es evidente, pues, que la escuela tiene una gran responsabilidad a la hora de forjar lectores competentes. Así, nos centraremos en estas páginas en los procesos de formación —inicial y permanente — del profesorado, tomando como referencia la UCA y el CEP de Cádiz.

El principal objetivo es mostrar nuestro trabajo y aportar ideas que potencien una reflexión de cara a adoptar medidas para solucionar este escollo que nos ocupa y preocupa. De acuerdo con los versos de Benedetti, nuestra actitud ante la formación lectora de la ciudadanía pasa por una negativa rotunda a la rendición: cada medida encaminada a forjar nuevos lectores suma.

\section{Caminante, no hay camino, se hace camino al andar (o el camino hacia la profesión docente)}

Comenzamos este apartado con las palabras de Machado extraídas de "Proverbios y cantares XXIX", puesto que partimos del convencimiento de que un docente conoce el momento en que se inicia su proceso formativo, pero será la propia actividad profesional la que trace la senda que habrá de seguir en su avatar diario. En este sentido, compartimos la preocupación de aquellos investigadores que, como Imbernón (1989), vienen reclamando desde hace algún tiempo un cambio de modelo en el que la formación inicial posibilite un análisis global de las situaciones pedagógicas y la permanente se apoye en el análisis, la reflexión, la investigación y la intervención docente del profesorado en ejercicio. 
Al menos en el panorama legislativo se están reflejando los reclamos de la comunidad investigadora. Puesto que focalizaremos nuestra atención en instituciones andaluzas la UCA y el CEP de Cádiz - analizaremos muy someramente la legislación que regula la formación del profesorado en esta comunidad autónoma. Como se expone en el Decreto 93/2013, de 27 de agosto por el que se regula la formación inicial y permanente del profesorado en la Comunidad Autónoma de Andalucía, así como el Sistema Andaluz de Formación Permanente del Profesorado:

\begin{abstract}
La formación del profesorado constituye un elemento fundamental para dar respuesta a los nuevos retos educativos que plantea la sociedad actual, siendo el factor clave para conseguir la mejora de la competencia profesional de los docentes y contribuyendo, en consecuencia, al desarrollo de una enseñanza de calidad y equidad. En este sentido, diversos informes de la Unión Europea, así como investigaciones y estudios específicos, ponen de manifiesto una clara correlación entre la preparación del profesorado y un sistema educativo de calidad, estableciendo vínculos significativos entre los programas de formación del profesorado, la mejora de la educación y los resultados del aprendizaje del alumnado.
\end{abstract}

Además, para la Ley 17/2007, de 10 de diciembre, de Educación de Andalucía, la esencia de la formación inicial se sustenta en la relación entre la teoría y la práctica y en la preparación para dinamizar los procesos de enseñanza y aprendizaje y de desarrollo personal del alumnado. En cuanto a la formación permanente, se determina que debe dar respuesta a las líneas estratégicas del sistema educativo, a las necesidades demandadas por los centros y al diagnóstico de necesidades que se desprendan de los planes de evaluación desarrollados.

Las líneas estratégicas vienen determinadas por la Orden de 31 de julio de 2014, por la que se aprueba el III Plan Andaluz de Formación Permanente del Profesorado y suponen el punto de partida para que los centros del profesorado de esta comunidad autónoma puedan dar respuesta a las necesidades de una sociedad cada vez más exigente en lo que a educación se refiere. Estas cinco líneas quedan reflejadas en la FIGURA 1.

\title{
LÍNEAS ESTRATÉGICAS
}

III PLAN ANDALUZ DE FORMACIÓN PERMANENTE DEL PROFESORADO

LÍNEA 1: Mejora de las prácticas educativas, el rendimiento y el éxito educativo.

LÍNEA 2: Perfeccionamiento continuo y capacitación profesional.

LÍNEA 3: Conocimiento compartido, investigación e innovación.

LÍNEA 4: Centros educativos como entornos colaborativos de aprendizaje y formación.

LÍNEA 5: Formación Profesional, Enseñanzas Artísticas, Enseñanzas Oficiales de Idiomas y Educación Permanente.

Figura 1: Líneas estratégicas para la formación del profesorado en Andalucía 
Como se aprecia, la formación inicial y la permanente del profesorado están estrechamente relacionadas ya que constituyen dos etapas de un mismo recorrido. Si se pretende lograr el éxito educativo, tanto las universidades como los centros de formación del profesorado deben caminar en la misma dirección. Esta idea recuerda a lo planteado por Zeichner (2010) al reivindicar un tercer espacio donde confluya lo que se enseña en las facultades y lo que se practica en los centros escolares. En este sentido, las asignaturas se plantean como una oportunidad profesionalizante en la que los estudiantes tienen la opción de integrarse de pleno en la vida de los centros educativos, experimentar -0 simular - los procesos de enseñanza y aprendizaje, analizar las necesidades formativas y estudiar los resultados de las pruebas externas ${ }^{7}$.

\section{Educar es lo mismo que poner motor a una barca (o la formación inicial)}

Para dirigir nuestra mirada hacia la formación inicial del profesorado partimos de la metáfora que nos ofrece Gabriel Celaya en su poema "Educar": si no se cuenta con las herramientas necesarias para enseñar ¿cómo hacerlo entonces? Como hemos expuesto en el apartado anterior, concebimos la formación inicial del profesorado como el inicio de un camino que habrá de completarse con los años de ejercicio de la profesión.

Somos conscientes de que la formación de un docente comporta multitud de aspectos que serían inabarcables y de que cada universidad tiene cierto margen de autonomía para definir su itinerario formativo siempre que se respeten las competencias generales de los títulos ofertados. De este modo, nos limitaremos a analizar la inclusión de asignaturas y líneas de investigación destinadas a forjar jóvenes lectores en el plan de estudios de la UCA. Para ello será preciso atender a las asignaturas de los Grados de Magisterio en Educación Infantil y Primaria; al Máster Universitario en Investigación para el Desarrollo Profesional Docente; y al Máster Universitario en Profesorado de Educación Secundaria Obligatoria y Bachillerato, Formación Profesional y Enseñanza de Idiomas. A estas asignaturas añadiremos las líneas de investigación ofertadas por el Departamento de Didáctica de la Lengua y la Literatura en la Escuela de Doctorado de la UCA (en adelante, EDUCA).

Como desgrana Ballester (2015) al hacer una retrospectiva histórica sobre la presencia de la literatura infantil y juvenil en la formación docente, los planes de estudio de las universidades españolas están aumentando progresivamente el número de asignaturas dedicadas a la formación de jóvenes lectores, aunque destaca especialmente su presencia en programas de máster, posgrados y cursos de formación continua. Además, como señalan Moreno y Sánchez (2000), la adscripción general de la docencia de esta materia se sitúa en el área de Didáctica de la Lengua y la Literatura, cuestión que ha sido determinante para trazar líneas de investigación específicas de la didáctica de la literatura y que está contribuyendo notablemente a aumentar el número de investigaciones en este ámbito.

\footnotetext{
7 Actualmente solo se realizan pruebas externas en Educación Primaria, las pruebas ESCALA, en segundo curso. De igual modo, pueden interpretarse como pruebas externas los informes realizados por el Servicio de Inspección Educativa tras una intervención en los Factores Clave para el funcionamiento de los centros escolares.
} 


\section{Grado de Magisterio en Educación Infantil}

Todo estudiante que se esté formando para ejercer como docente en Educación Infantil debe estar familiarizado con la literatura infantil, las características del lector al que va dirigida, sus señas de identidad, las opciones para fomentar la lectura... En la UCA, a lo largo de los estudios del Grado se hace constante referencia a la literatura infantil, especialmente en las asignaturas vinculadas al Departamento de Didáctica de la Lengua y la Literatura y en las fases prácticas, pero no es hasta el octavo semestre cuando se imparte la asignatura obligatoria 41118024 Literatura Infantil y Fomento de la Lectura, de 6 créditos. En la FIGURA 2 se exponen los contenidos abordados y los resultados de aprendizaje esperados tras cursar la asignatura:

\section{Literatura Infantil y Fomento de la Lectura}

Contenidos:

- Delimitación del concepto de Literatura Infantil.

- El corpus la Literatura Infantil y su funcionalidad.

- La Literatura Infantil en el currículo de Educación Infantil.

- El fomento de la lectura en Educación Infantil.

- Literatura Infantil en lengua materna.

- Literatura Infantil y multiculturalidad.

Resultados de aprendizaje:

1. Analizar y valorar las producciones de la tradición oral y su relación con los hábitos lectores y la competencia literaria.

2. Valorar la literatura como un elemento formativo y transmisor que engloba valores universales como los principios de igualdad, respeto y cultura de paz desde una perspectiva multicultural.

3. Conocer y aplicar diferentes técnicas de promover la lectura en el aula y la formación de hábitos lectores.

4. Saber elaborar una selección de obras apropiadas para el trabajo en el aula.

5. Conocer las características de la literatura infantil y relacionarla con el currículo de Educación Infantil.

6. Conocer y analizar el corpus literario de la literatura infantil.

Figura 2: Literatura infantil y fomento de la lectura. Grado de Magisterio en Educación Infantil [Para mayor información, consúltese el siguiente enlace: https://goo.gl/iIFFVE]

\section{Grado de Magisterio en Educación Primaria}

El caso de la presencia de la literatura infantil y el fomento lector no es diferente en el Grado de Magisterio en Educación Primaria. Las referencias son constantes tanto en las asignaturas como en las experiencias prácticas. No obstante, este Grado cuenta con dos asignaturas dedicadas específicamente a esta materia. La primera, 41119024 Infantil y Fomento de la Lectura, de 6 créditos, tiene un carácter obligatorio y se imparte en el quinto semestre; la segunda, 41119045 La Literatura Infantil como experiencia 
plurilingüe, también de 6 créditos, es optativa y se inserta dentro de la mención AICLE ofertada en esta universidad. Está programada para el octavo semestre. En las FIGURAS 3 y 4 se exponen los contenidos y resultados de aprendizaje de ambas asignaturas.

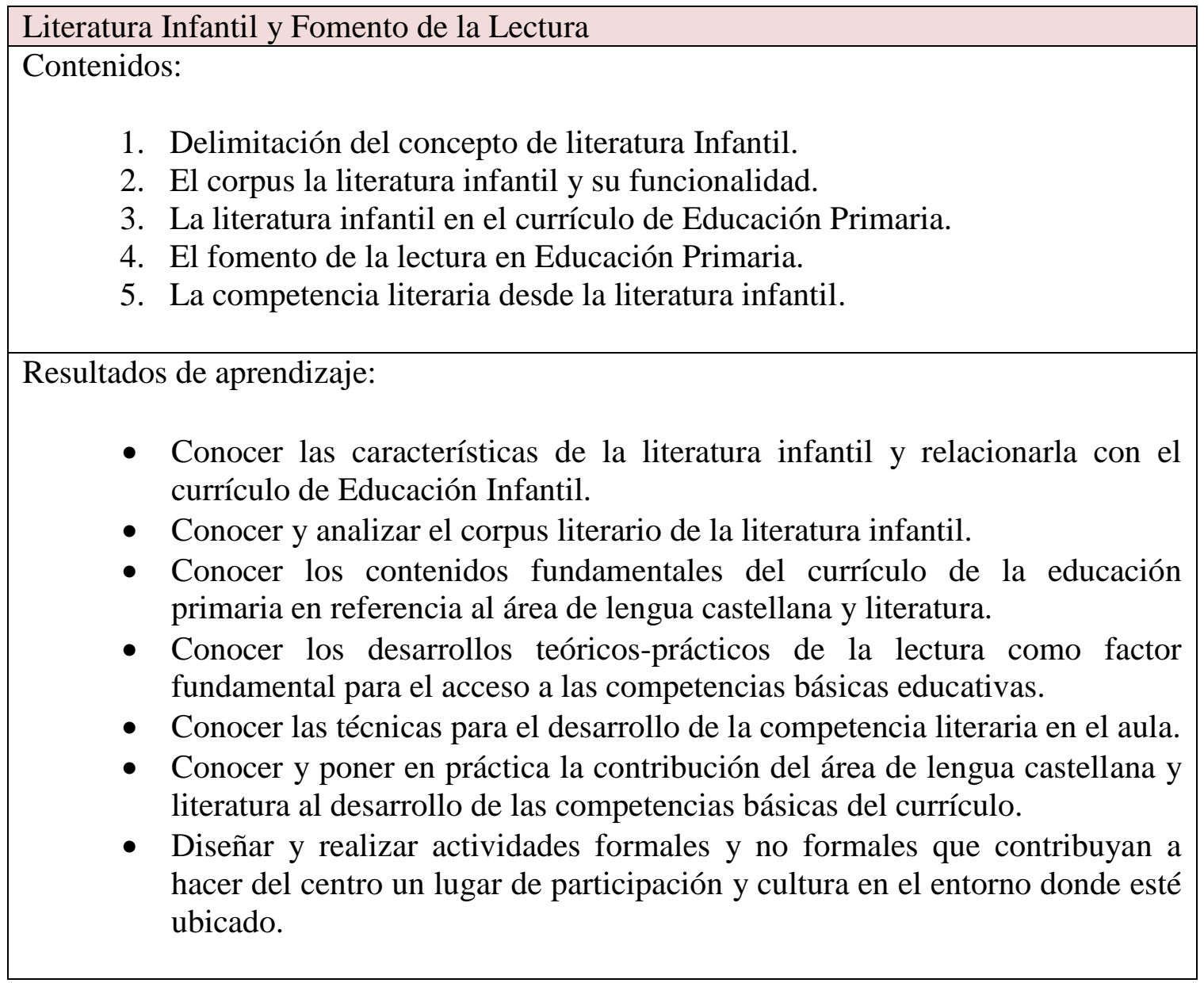

Figura 3: Literatura infantil y fomento de la lectura. grado de Magisterio en Educación Primaria [Para mayor información consúltese: https://goo.gl/Njgpzi]

\section{La Literatura Infantil como experiencia plurilingüe}

Contenidos:

1. Introducción al enfoque metodológico AICLE y al desarrollo de la educación plurilingüe y pluricultural en Educación Primaria.

2. La literatura infantil en LE. Principales aportaciones.

3. Técnicas y recursos para trabajar la Literatura Infantil en el proceso de e/a en L2, L3, L4 en Educación Primaria.

4. La competencia literaria a través de la literatura y el folclore infantil en L2, L3, L4. La dimensión intercultural en la literatura infantil.

Resultados de aprendizaje:

- Analizar y entender las ventajas de la integración de contenidos literarios y 
lenguas extranjeras en la educación plurilingüe y pluricultural en Educación Primaria.

- Conocer los procedimientos para integrar la literatura infantil en la enseñanza/aprendizaje de idiomas en la Educación Primaria.

- Diseñar propuestas didácticas para trabajar la literatura infantil en la enseñanza/aprendizaje de LE.

- Conocer las principales aportaciones de la literatura infantil en LE.

- Tomar conciencia de la dimensión intercultural de la literatura infantil.

Figura 4: La literatura infantil como experiencia plurilingüe

Como se aprecia en los contenidos y en los resultados de aprendizaje esperados tras cursar las asignaturas citadas, en todo momento se busca una relación entre la teoría y la práctica y se estimula la experimentación o simulación de situaciones de enseñanza y aprendizaje en un camino que se inicia con los conceptos teóricos, pasa por lo prescrito en la legislación educativa en vigor y culmina en las situaciones "reales" de aula.

Además, el carácter obligatorio de una asignatura en los Grados de Magisterio dedicada específicamente a la literatura infantil y al fomento de la lectura garantiza que los docentes - tras su período de formación inicial — posean una formación básica para seleccionar obras adecuadas al público infantil y, por ende, ejercer el papel de mediadores en la lectura cumpliendo con las condiciones propuestas por Cerrillo, Larrañaga \& Yubero (2002). No obstante, será la formación permanente la encargada de afianzar estos conocimientos y actualizar didácticamente a los docentes en ejercicio para que, paulatinamente, puedan experimentar un verdadero desarrollo profesional.

\section{Máster Universitario en Investigación para el Desarrollo Profesional Docente}

Este máster se compone de 60 créditos, repartidos en dos módulos, uno por cada semestre. El primer semestre se dedica al módulo fundamental y el segundo al módulo de formación complementaria. Atendiendo a la formación de lectores competentes, encontramos tres asignaturas relacionadas directamente con este ámbito:

a. Una obligatoria para todo el alumnado, perteneciente al módulo fundamental: 1170002, Problemáticas relativas a la competencia en la comunicación lingüística y la interacción del conocimiento en el currículum escolar, de 4 créditos. Esta asignatura se vincula directamente con la vida de los centros escolares andaluces ya que se atiende fundamentalmente al diseño y puesta en marcha de los Proyectos Linguísticos de Centro que cuentan, entre sus líneas de actuación, con el fomento lector y la dinamización de las bibliotecas escolares ${ }^{8}$.

b. Y dos optativas, de 3 créditos cada una, integradas dentro del módulo de formación complementaria que complementan a la asignatura obligatoria ya que responden a los dos pilares esenciales para formar jóvenes lectores: 1170106 La formación del lector y 1170107 Perspectivas de investigación en escritura en contextos educativos. De nuevo, estas dos asignaturas se relacionan directamente con la realidad vivida en los centros educativos andaluces ya que se muestran las posibilidades de formación permanente que ofrece la Consejería de Educación de la Junta de Andalucía (en adelante, CEJA).

\footnotetext{
${ }^{8} \mathrm{Si}$ se desea obtener más información sobre estos proyectos, puede consultarse el portal diseñado ad hoc: https://goo.gl/OwDmQh.
} 


\section{Máster Universitario en Profesorado de Educación Secundaria Obligatoria y Bachillerato, Formación Profesional y Enseñanza de Idiomas}

Tal y como se refleja en la unidad de posgrado de la UCA, este máster pretende aportar una formación inicial para el desarrollo de la función docente, integrada por conocimientos teóricos, habilidades, actitudes y un contacto suficiente con la profesión y su práctica. Además, a partir de la oferta de asignaturas optativas, se puede obtener una formación básica sobre investigación educativa en un área disciplinar concreta ya que está estrechamente conectada con las líneas de investigación sobre educación ofertadas por los distintos departamentos de la Facultad de Ciencias de la Educación.

Igual que el máster de investigación, consta de 60 créditos, repartidos en cuatro módulos: módulo común, módulo específico, módulo transversal y módulo de aplicación. En lo concerniente a la formación de lectores competentes, contamos con tres asignaturas, todas obligatorias, dentro del módulo específico del área de enseñanza de lenguas:

- Complementos de formación en la especialidad, de 6 créditos. En esta asignatura, entre otros contenidos se analizan las características de la literatura de la adolescencia y la juventud, se da a conocer el corpus de lecturas de la adolescencia y la juventud, se aportan estrategias para desarrollar un adecuado fomento de la lectura y se analizan las conexiones entre la literatura y la imagen.

- Aprendizaje y enseñanza en la especialidad, de 12 créditos. En esta asignatura se aborda específicamente la didáctica de la literatura, sus enfoques metodológicos, recursos y estrategias para simular situaciones de enseñanza y aprendizaje que luego los estudiantes habrán de experimentar en el módulo de aplicación.

- Innovación docente e iniciación a la investigación en el ámbito de especialización, de 6 créditos. Esta asignatura se vincula especialmente con los planes y programas que la CEJA pone a disposición del profesorado para potenciar la innovación docente. Además de ello, se realiza un acercamiento a las Bibliotecas Escolares Como Recurso de Enseñanza y Aprendizaje (en adelante, BECREA).

\section{La EDUCA}

La UCA cuenta con una escuela de doctorado que oferta diez programas diferentes. El que atiende a la formación de lectores es el de Ciencias Sociales y Jurídicas, concretamente a través de la línea de investigación Educación y Formación del Profesorado. Dentro de esta línea de investigación, el Departamento de Didáctica de la Lengua y la Literatura ofrece la posibilidad de profundizar en la formación recibida tanto en el Máster Universitario en Profesorado de Enseñanza Secundaria Obligatoria y Bachillerato, Formación Profesional y Enseñanza de Idiomas como en el Máster Universitario en Investigación para el Desarrollo Profesional Docente y con ello dar respuesta a las demandas que plantea la educación en los diferentes niveles educativos.

Este departamento comparte las ideas de Mendoza (2003) al plantear la investigación en Didáctica de la Lengua y la Literatura desde grandes ámbitos referidos a la metodología, los procesos, los contextos, los contenidos y las creencias. Estos presupuestos se concretan en las siguientes líneas de investigación, vinculadas al grupo PAI HUM748 Grupo para el estudio de la enseñanza de la lengua y la literatura: 
1. Investigación-acción en enseñanza-aprendizaje de la lengua oral y escrita (L1, L2) en infantil/primaria/secundaria/Escuelas Oficiales de Idiomas (EOI).

2. Enfoques de la lectura y la escritura en el currículum.

3. Educación literaria: fomento lector y literatura infantil y juvenil (L1, L2): teoría, crítica y su didáctica.

4. Enseñanza y aprendizaje de la gramática.

5. Competencia sociolingüística en el aprendizaje de lenguas.

6. Didáctica del léxico.

7. Disponibilidad léxica y desarrollo de las habilidades comunicativas.

8. Escritura académica.

9. Plurilingüismo y Aprendizaje Integrado de Contenidos en Lengua Extranjera (AICLE).

Como se aprecia, tras estudiar el plan de estudios de la UCA — Grados de Magisterio, Másteres y Programa de Doctorado-, podemos afirmar que la presencia de la literatura infantil y juvenil en la formación inicial docente es cada vez mayor. Se han consolidado asignaturas específicas en los Grados de Magisterio y en los Másteres de acceso inmediato para los graduados. Además, el hecho de que estas asignaturas supongan un paso obligatorio para la obtención del título supone un paso a favor de la equidad en la preparación y del conocimiento de las lecturas que habrán de fomentarse en las escuelas. Aun así, esta formación es solo el inicio del camino hacia la configuración de la identidad docente.

\section{¡Empuña el libro, hambriento! ¡Es un arma! (o la formación permanente)}

Desde nuestro punto de vista, la formación permanente del profesorado debería seguir un proceso análogo a lo expresado en los versos de Bertolt Brecht en su "Elogio al aprendizaje", generando una suerte de hambre de conocimiento que comienza al inicio de la carrera profesional y que no habrá de terminar jamás.

Los objetivos de la formación permanente del profesorado son diversos, pero todos giran en torno al aumento de la calidad educativa ya sea contribuyendo al desarrollo profesional de los docentes; aportando estrategias útiles para resolver los problemas planteados en el aula; contribuyendo a la mejora de los centros escolares y, por extensión, a la mejora de los procesos aprendizajes del alumnado. Para lograr estos objetivos será necesario que se cumplan dos condiciones: un adecuado conocimiento de las demandas del profesorado y un correcto proceso de evaluación.

De acuerdo con esta idea está lo expuesto por Manzanares y Galván-Bovaira (2012, p. 435) al reivindicar la necesidad de traspasar la creencia de que lo aprendido en cursos, encuentros o congresos se aplica automáticamente al aula y de diseñar modelos de evaluación para que contribuyan a "profundizar tanto en aspectos conceptuales referentes a la FPP, como en aquellos elementos más prácticos conducentes a un mejor aprovechamiento de los recursos formativos".

Como indicábamos más arriba, en estas páginas vamos a centrarnos en los elementos que favorecen el aumento de la formación de los docentes andaluces en ejercicio a la 
hora de forjar jóvenes lectores. En este sentido, los primeros interrogantes que podrían surgir a un docente que desee actualizarse en esta materia girarían en torno a ¿cuáles son los cauces de formación existentes?; ¿qué posibilidades formativas se contemplan?; ¿qué modalidad formativa es más efectiva? Dado que las posibilidades son múltiples, vamos a tratar de organizar la información partiendo de nuestra experiencia como asesora de formación del ámbito linguístico del CEP de Cádiz en torno a tres bloques: la red profesional BECREA; los Planes y Programas de la CEJA y los cursos de formación ofertados por el CEP de Cádiz durante el año académico 2015-2016. No atendemos en este trabajo a las modalidades de autoformación - Grupos de Trabajo y Formación en Centros - puesto que las temáticas demandadas son tan variadas que nos alejarían del objetivo planteado.

\section{La red profesional BECREA}

Las redes profesionales provinciales de bibliotecas escolares son herramientas de información, interconexión, apoyo, comunicación, asesoramiento y cooperación entre el profesorado responsable de las bibliotecas de los centros educativos andaluces. Hacen posible la autoformación del profesorado y el enriquecimiento mutuo al compartir las experiencias vividas en los centros educativos.

Según lo dispuesto en las instrucciones de 24 de julio de 2013, de la Dirección General de Innovación Educativa y Formación del Profesorado sobre la Organización y funcionamiento de las bibliotecas escolares de los centros docentes públicos que imparten Educación Infantil, Educación Primaria y Educación Secundaria, cada docente responsable de la biblioteca escolar deberá incorporarse y participar como miembro efectivo en la red profesional de su provincia, dirigida por la Delegación Territorial con el apoyo de los Centros del Profesorado. Así, cada Delegación Territorial dispone de su propia plataforma virtual en la que se registran sus miembros.

En nuestra opinión, la creación de estas redes profesionales está facilitando una formación directa y efectiva, a medida de los profesionales que la utilizan para desarrollar su labor como responsables de las bibliotecas escolares. Es una oportunidad de desarrollo profesional compartido y cuentan con el apoyo de docentes expertos en activo que dinamizan la red y resuelven las dudas planteadas a diario.

\section{Los Planes y Programas de la CEJA}

Cada año, la Dirección General de Innovación de la CEJA ofrece la oportunidad a los centros escolares sostenidos con fondos públicos de que se inscriban en Planes o Programas que favorezcan la innovación educativa y la paulatina transformación de los centros. Estos Planes y Programas cuentan con el apoyo formativo de los Centros del Profesorado que se encargan de diseñar la formación necesaria para los centros escolares que se inscriben. Existen tres tipos de programa, según la duración y el nivel de implicación de profesorado requerido, como se expresa en la FIGURA 5: P1, P2 Y P3. 


\begin{tabular}{|l|l|}
\hline \multicolumn{2}{|c|}{ TIPOLOGÍA DE PLANES Y PROGRAMAS OFERTADOS POR LA CEJA } \\
\hline P1 & $\begin{array}{l}\text { Tienen una duración de tres cursos escolares, exigen la participación de, al } \\
\text { menos, el cincuenta por ciento profesorado del claustro e incluyen una } \\
\text { formación específica adaptada a las necesidades de los centros escolares } \\
\text { según las líneas de actuación que deseen desarrollar cada año }\end{array}$ \\
\hline P2 & $\begin{array}{l}\text { Tienen una duración de al menos un curso escolar, precisan de la } \\
\text { participación de, al menos, el diez por ciento del profesorado del claustro e } \\
\text { incluyen una formación para la persona coordinadora. }\end{array}$ \\
\hline P3 & $\begin{array}{l}\text { Tienen una duración de un curso escolar, el profesorado puede participar de } \\
\text { forma individual y no incluyen una formación específica, tan solo es } \\
\text { obligatoria la asistencia a una jornada informativa para poner en marcha la } \\
\text { actividad. }\end{array}$ \\
\hline
\end{tabular}

Figura 5: Características de los Planes y Programas de la CEJA según su tipología

Todos los Planes y Programas, independientemente de su rango, cuentan con una plataforma virtual de apoyo para compartir experiencias, materiales, inquietudes... Como elementos de innovación de los centros escolares, deben incluirse en los Planes de Centro e informarse de su participación al Consejo Escolar.

Dentro del ámbito que nos ocupa, el lingüístico, se ofertan cinco programas, un P1: el Proyecto Linguiístico de Centro (en adelante, PLC); y cuatro P2: Clásicos Escolares; ComunicAcción; Familias Lectoras y Creatividad Literaria. Aunque sin entrar en profundidades, consideramos que es preciso explicar en qué consiste cada programa.

\subsection{Proyecto Lingüístico de Centro}

El PLC tiene como objetivo impulsar y apoyar la puesta en marcha en los centros de propuestas encaminadas a la mejora de la competencia linguiística tanto desde las áreas lingüísticas como desde las áreas no lingüísticas.

Por tanto, se trata de una medida de actuación global en cuyo horizonte se encuentra la innovación educativa y la renovación metodológica compartida por todo el profesorado del centro que, a través de la coordinación de aquellas actuaciones que desarrollan la competencia lingüística, irá mejorando paulatinamente las habilidades orales y escritas del alumnado.

Las principales líneas de actuación de un PLC persiguen la mejora de la competencia comunicativa del alumnado y se insertan en distintos ámbitos: regulación de aspectos normativos relacionados con los usos lingüísticos en el centro - cuestión que permite una mejora sustancial en la convivencia-, tratamiento de la diversidad lingüística, medidas de coordinación y apoyo al proyecto de plurilingüismo - Enfoque AICLE y CIL-, elaboración de un plan integral de mejora de la competencia lingüística en lengua materna - atendiendo a las cinco destrezas-, diseño del plan lector y del plan de uso de la biblioteca escolar del centro.

Como se aprecia en la FIGURA 5, se trata de un P1. Esto conlleva la exigencia de un alto grado de implicación de los centros participantes. Se precisará de una efectiva 
coordinación y una adecuada respuesta por parte del profesorado. No obstante, para lograr el éxito en el desarrollo del programa, será necesario el apoyo de los Centros del Profesorado con el diseño de un plan de formación en el que será indispensable la participación de la persona coordinadora y, en distintos momentos, del profesorado que forme parte del equipo de trabajo.

\subsection{Clásicos Escolares}

El objetivo del programa Clásicos Escolares es ofrecer estrategias y recursos metodológicos que puedan complementar el currículum escolar. En este sentido, se aporta una visión integradora de la literatura clásica como manifestación cultural, lingüística, estética, social, ética e individual que se vincula con las competencias clave y las nuevas metodologías educativas.

Para logarlo, se propone una metodología basada en la riqueza de los textos clásicos y la participación activa de los estudiantes como lectores en formación. Además, se apoya al profesorado para el diseño de actividades lectoras centradas en los textos clásicos y en el entramado de referencias que encierran estas creaciones. Para su desarrollo, el programa cuenta con tres líneas de actuación:

- Clásicos andaluces, para incentivar la lectura de los textos escritos por autores andaluces.

- Clásicos hispánicos e hispanoamericanos, cuya pretensión principal es la lectura de los textos de autores que conforman la historia de la literatura escrita en español y de todas las realidades mencionadas en ellos.

- Clásicos universales, cuyo propósito es la lectura de las obras que han terminado por ofrecer tópicos, obras o manifestaciones artísticas atemporales, en diversas lenguas, que todavía hoy son revividas en nuestra sociedad. Así, cabe la posibilidad de desarrollar actividades integradas por distintas lenguas y materias, pudiendo sobrepasar los límites de la lengua y la cultura española.

\subsection{ComunicAcción}

La participación en este programa supone aceptar el reto de integrar en el aula la metodología de Aprendizaje Basado en Proyectos para trabajar los medios audiovisuales en el aula, desarrollando las competencias clave y obteniendo un producto final. Para ello, será necesaria la puesta en marcha de acciones de comunicación que doten al alumnado de autonomía para emprender proyectos relacionados con los medios de comunicación.

Mediante la creación de un medio de comunicación se favorecerá el desarrollo la competencia en lingüística, el uso adecuado y reflexivo de las TIC, el conocimiento del periodismo, del diseño, del arte y la comunicación en general.

Cada centro puede desarrollar este programa de la forma que mejor se ajuste a sus necesidades y características, bien de forma interdisciplinar o bien como temática independiente. No obstante, este programa ofrece a los docentes y a los estudiantes una herramienta eficaz e integradora para trabajar todas las competencias clave desde todas las áreas del currículo. Además, los centros cuentan con en apoyo necesario para 
generar recursos digitales y audiovisuales para difundir sus prácticas docentes $\mathrm{o}$ experiencias de interés.

\subsection{Familias Lectoras}

El principal objetivo de este programa es ayudar a las familias en su labor educadora, de modo que, en colaboración con el centro educativo, les oriente y anime a despertar en sus hijos, la afición por el libro, a mejorar su comprensión lectora y a afianzar el hábito de la lectura.

Dado que nos enfrentamos a un reto complejo, con la implicación de las familias se pretende abrir el apetito lector del alumnado, fomentar el trabajo cooperativo de todos los estamentos del centro en diversas actividades e inculcar a toda la comunidad educativa la pasión por los libros. No siempre se recogen los frutos deseados, pero no corren tiempos para la rendición, hay que seguir sembrando en estas etapas educativas esenciales para la creación del hábito lector y, en definitiva, para forjar futuros lectores.

Este programa cuenta con el portal "Familias lectoras en red", un espacio de encuentro e intercambio para familias, profesorado y otro personal educativo de todo el territorio nacional. En este portal se muestran buenas prácticas relacionadas con la lectura y la escritura desarrolladas por los centros escolares, las AMPAS y otras instituciones implicadas - Ayuntamientos, bibliotecas públicas, ONG, fundaciones, etc.- .

\subsection{Creatividad Literaria}

El principal objetivo de este programa es lograr que el alumnado realice una reflexión personal y crítica sobre su tradición literaria, impulsando la creación de textos, como modo de construcción del propio pensamiento, de expresión personal y de goce.

Para plantear actividades creativas es necesario modificar los procesos de enseñanza y aprendizaje, abriendo en el aula espacios de expresión y dando validez a las diferentes respuestas y soluciones aportadas por el alumnado. En este sentido, la lectura y la escritura supondrán un estímulo creador para jugar con las palabras, revalorizarlas, redescubrirlas, hacerlas propias y conectar con ellas para expresarse uno mismo.

Cada centro tiene la posibilidad de desarrollar el programa de la forma que mejor se ajuste a sus necesidades y características, ya sea de forma interdisciplinar, como apoyo al currículum en el aula, desde la biblioteca escolar, o bien como temática independiente.

3. Cursos de formación ofertados por el CEP de Cádiz durante el año académico 2015-2016.

El Real Decreto 2112/1984 de 14 de noviembre concibe los Centros de Profesores como "instrumentos preferentes para el perfeccionamiento del profesorado y el fomento de su profesionalidad, así como para el desarrollo de actividades de renovación pedagógica y difusión de experiencias educativas". Aunque las distintas comunidades autónomas hayan ido ajustando la labor de estos centros, otorgándoles distintas competencias e 
incluso prescindiendo de ellos, para la CEJA, los Centros de Profesorado siguen teniendo la esencia anglosajona de los Teachers' Centers. En nuestra comunidad autónoma, los CEP son la piedra angular de la formación permanente del profesorado y su red de asesorías - que cuenta con más de 300 miembros - se encarga de impulsar, dinamizar y acompañar al profesorado andaluz en sus procesos de formación, reflexión e investigación. Nuestra red de formación incluye asesorías dedicadas a la Formación Profesional, a la Educación Permanente, Infantil, Primaria y Secundaria. Dentro de la etapa secundaria, y con el fin de atender de manera efectiva a las didácticas específicas, las asesorías se agrupan en cuatro ámbitos: el ámbito linguístico, el ámbito científico, el ámbito social y cívico y el ámbito artístico y deportivo.

Nuestra labor en el CEP de Cádiz se adscribe al ámbito lingüístico, principalmente al trabajo en la Didáctica de la Lengua y la Literatura de la lengua materna. Existe otra asesoría que se encarga de las lenguas extranjeras y de las Escuelas Oficiales de Idiomas.

La experiencia del CEP de Cádiz para abordar la formación complementaria en didácticas específicas nos hizo dirigir los esfuerzos hacia la creación de un itinerario de apoyo a los programas de nuestro ámbito. Aún no disponemos de los datos suficientes como para calibrar el impacto que esta formación ha tenido en el aula, pero sí podemos afirmar que el nivel de satisfacción de ponentes y asistentes ha sido muy alto.

En este trabajo realizaremos un breve recorrido por las actividades formativas de carácter abierto, para todo el profesorado que las precisó, organizadas por nuestro CEP el pasado curso 2015/2016 por si sirven de inspiración para otras asesorías de formación o despiertan interés por su participación para el profesorado.

\subsection{Hacia la elaboración de un PLC desde las áreas lingüísticas y no lingüísticas}

Esta acción formativa consistió en un curso presencial, de 15 horas, impartido en el mes de octubre de 2015 por Raúl Rubio Millares, profesor de Lengua Castellana y Literatura en Educación Secundaria, agente clave en la trasformación del IES García Lorca de Algeciras (Cádiz) por medio de la implantación de un PLC. Estaba dirigido al profesorado de las etapas Primaria y Secundaria que se planteaba elaborar un proyecto lingüístico de centro. En sus sesiones se conceptualizó el PLC, se aportaron modelos, se mostró cómo elaborar un PLC desde una perspectiva sistémica, se enseñaron estrategias para la difusión del proyecto y, por último, se elaboró, en pequeños grupos, un proyecto tipo.

A partir de su celebración, se crearon varios grupos de trabajo encaminados a la preparación de un futuro PLC en cuatro centros escolares que aún no se habían inscrito al programa. Podemos afirmar, pues, que supuso un primer impulso para continuar el proceso de formación permanente, derivando hacia otras modalidades de autoformación que están impregnando paulatinamente la vida de los centros de nuestro ámbito de actuación.

\subsection{Literatura juvenil y nuevas tecnologías}


Este curso, de 15 horas presenciales y 15 no presenciales, impartido por Noemí Infantes Moreiras, responsable de la biblioteca escolar del IES Virgen del Carmen de Puerto Real (Cádiz), en el mes de noviembre de 2015, propició el acercamiento de los docentes a la literatura juvenil actual. Además, logró fomentar la reflexión y el análisis de los métodos tradicionales de fomento lector. Así, se discutieron los puntos primordiales relacionados con el tratamiento de la lectura, desde el planteamiento de lecturas obligadas o recomendadas, clásicas o juveniles - hasta la elaboración de un plan lector de centro.

La metodología fue principalmente práctica, con posibilidades de aplicación inmediata en el aula puesto que lo que se pretendía era compartir experiencias y dotar al profesorado asistente de herramientas útiles para trabajar la lectura en el contexto

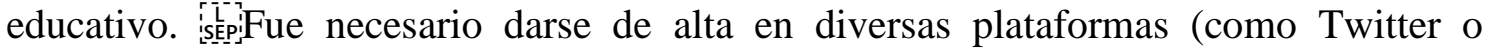
Padlet) pero no se precisaron grandes conocimientos informáticos. Las horas no presenciales se destinaron a la confección de una lista de lecturas recomendadas, a la lectura - y posterior puesta en común - de dos novelas juveniles y a la elaboración de un Padlet colaborativo.

\subsection{La biblioteca escolar, entorno de aprendizaje y lectura}

Esta actividad formativa, de 15 horas presenciales, se desarrolló durante el mes de enero de 2016 y fue impartida por Andrés Pulido Villar, profesor de Lengua Castellana y Literatura en Educación Secundaria y dinamizador de la Red Profesional de Bibliotecas escolares en la provincia de Cádiz.

La biblioteca escolar es un recurso educativo al servicio del currículum y el proyecto educativo del centro y se concibe en la actualidad como un espacio dinámico de recursos y servicios de información que cumple un papel primordial en el proceso de enseñanza y aprendizaje, y como tal es un instrumento fundamental de índole pedagógica que apoya la labor docente.

En consonancia con este marco conceptual, las cuatro primeras sesiones del curso se dedicaron a exponer las principales líneas de actuación de la BECREA en este ámbito, a conocer los recursos necesarios y a analizar modelos ilustrativos para que la biblioteca escolar pudiera contribuir al desarrollo del proyecto educativo y explotar sus posibilidades didácticas. Asimismo, a lo largo de estas sesiones, se trabajó en varias propuestas prácticas a partir de los contenidos marcados. En la quinta y última sesión se organizó un encuentro con el escritor Eliacer Cansino, autor muy galardonado en el panorama nacional e internacional, entre cuyos premios destaca el Nacional de Literatura Infantil y Juvenil. Para finalizar, se contó con una mesa redonda en la que participaron los agentes implicados en las bibliotecas: un responsable de biblioteca escolar, una librera y el escritor Eliacer Cansino.

\subsection{Técnicas de creación literaria y oralidad}

Este curso, de 15 horas presenciales, tuvo lugar entre los meses de febrero y marzo de 2016 y fue impartido por Alberto Corpas Martos y por Pablo Moreno Verdulla. Se trató de una actividad formativa fundamentalmente práctica que dotó al profesorado de una 
serie de estrategias para trabajar de manera efectiva la expresión oral y escrita. Las sesiones se plantearon en forma de talleres dedicados al manejo de recursos y herramientas para ahondar en la creatividad del alumnado. Se aportaron ejemplos reales de aula como el calcetín rojo, el tendedero literario, el final alternativo, el voyeur, la imitatio, la actualización del clásico, el pechá kuchá, el kamishibai, la lectura compartida, el debate... Ambos ponentes son profesores Educación Secundaria de centros públicos de Andalucía especialistas en Lengua y Literatura con buenas prácticas de aula contrastadas.

\subsection{Ciclo de conferencias en torno a la enseñanza de la lengua y la literatura}

Esta actividad, que cerraba el itinerario en el mes de abril de 2016, englobó un ciclo de conferencias en torno a la enseñanza de la lengua y la literatura. Estaba dirigida al profesorado de Educación Infantil, Primaria, Secundaria y Bachillerato interesado en actualizar sus competencias docentes para desarrollar los aprendizajes lingüísticos y literarios de sus estudiantes. Partimos de la premisa de que la consolidación de la competencia en comunicación lingüística era clave para lograr el éxito académico. En este sentido, se organizaron cinco conferencias, cada una dedicada a un ámbito de la Didáctica de la Lengua y la Literatura:

a) El empleo de las TIC para el aprendizaje: "Itinerarios didácticos en la Didáctica de la Lengua y la Literatura", a cargo de José Antonio Hernández Ortega (Pep Hernández), profesor de Lengua Castellana y Literatura, investigador en Didáctica de la Lengua y la Literatura a través de las TIC, el aprendizaje cooperativo y las metodologías activas.

b) El aprendizaje temprano de la lectura y la escritura con carácter funcional: "Leer y escribir para vivir: aprendiendo/enseñando a leer y a escribir", ocasión en la que contamos con la presencia de Montserrat Fons Esteve, profesora del Departamento de Educación Lingüística y Literaria de la Universidad de Barcelona, especialista en alfabetización temprana, plurilingüismo en la escuela, creencias del profesorado y formación inicial y permanente.

c) La educación literaria: "Leyendo conjuntamente palabras, imágenes y sonidos con una mirada intertextual", de la mano de M. Alba Ambròs Pallarés, profesora Departamento de Educación Linguiística y Literaria de la Universidad de Barcelona, especialista en comunicación lingüística, lectura literaria, audiovisual y artística y la innovación docente en la formación del profesorado.

d) Las buenas prácticas en educación lingüística y literaria: "Buenas prácticas desde la experiencia: ¿Qué proponen los docentes experimentados?". Para esta jornada contamos con la presencia de Daniel Cassany Comas, profesor del Departamento de Traducción y Filología de la Universidad Pompeu Fabra de Barcelona cuya línea de investigación se centra en la comunicación escrita con diferentes propósitos, perspectivas y géneros. Actualmente dirige un proyecto sobre Literacidad Crítica.

e) El trabajo de la competencia en comunicación linguiística para el éxito 
escolar: "Leer, conversar y escribir en las escuelas desde las coordenadas espacio-tiempo". Esta conferencia final fue impartida por Manuel Francisco Romero Oliva, Director del Departamento de Didáctica de la Lengua y la Literatura de la Universidad de Cádiz, cuyas principales líneas de investigación son la escritura académica, la educación lingüística y literaria, la formación del profesorado y la enseñanza de español como segunda lengua.

Como se puede observar, en este ciclo de conferencias participaron especialistas con amplia experiencia procedentes de diversas universidades y centros educativos de todo el país. El nivel de satisfacción del profesorado asistente, dada la calidad de las conferencias, fue notable.

\section{El lunar es el punto final del poema de la belleza (o conclusión)}

Al inicio de este trabajo aludíamos a la preocupación social sobre el acercamiento a la lectura a los jóvenes de hoy, pasábamos de puntillas por los agentes encargados de formar a lectores competentes para centrarnos definitivamente en la responsabilidad que tiene la escuela en esta materia.

Dado que, desde nuestro punto de vista, el profesorado es un elemento importante en el éxito de los procesos de enseñanza y aprendizaje y que una adecuada formación - tanto inicial como permanente- puede ser determinante en la buena marcha de un sistema educativo, aprovechamos los dos frentes profesionales por los que transitamos para mostrar - y demostrar - que al menos las instituciones encargadas de formar a docentes están iniciando un camino para coordinar esfuerzos y navegar en la misma dirección. No obstante, debemos ser cautos a la hora de alzar las campanas al vuelo.

En lo concerniente a la formación inicial, responsabilidad en el caso que nos ocupa de la UCA, los planes de estudios que presentamos se han modificado recientemente y aún es escaso el número de egresados que se ha incorporado a la función docente.

En cuanto a la formación permanente, programada por el CEP de Cádiz en este estudio, aún solo contamos con los resultados de las encuestas de satisfacción del profesorado asistente y, aunque son muy positivos, debemos completar nuestra evaluación con el seguimiento del proceso de implementación en el aula. En la actualidad, estamos recabando información tratando de obtener evidencias, entrevistando a docentes que asistieron a la actividad, asesorándoles para poner en práctica lo aprendido en las acciones formativas... Auguramos que en un plazo de tres cursos tendremos resultados concluyentes.

A lo largo de estas líneas hemos trazado un camino similar al que podría realizar un docente, desde la formación inicial en el Grado de Magisterio hasta las posibilidades de formación permanente del profesorado. Para llegar al final de esta senda hemos transitado por la oferta de formación complementaria de la UCA y por las propuestas de innovación de la CEJA.

Aludiendo a Ramón Gómez de la Serna y a la greguería que da título a este apartado, 
ponemos nuestro lunar utilizando las palabras de Lluch (2010, p. 12):

\begin{abstract}
No podemos privar a las nuevas generaciones del placer que significa leer, de la misma manera que no les podemos privar del placer de pensar, porque ambos nos dan buena vida, se pueden lograr en soledad o en compañía, son baratos, te hacen reír y llorar, etc. Justamente, porque leemos y pensamos sabemos que la vida, la historia de nuestras vidas, es un fluir continuo de cambio y de renovación, pero siempre desde el conocimiento del pasado. Y todo eso requiere lecturas y requiere pensamiento. Y eso requiere tiempo.
\end{abstract}

\title{
Libros extraños que alagáis a la mente [Rubén Darío "libro extraño"] (o Referencias Bibliográficas)
}

Ballester, J. (2015). La formación lectora y literaria. Barcelona: Graó.

Barber, M. \& Mourshed, M. (2008). Cómo hicieron los sistemas educativos con mayor desempeño del mundo para alcanzar sus objetivos. Buenos Aires: Mckinsey \& Company.

Cerrillo, P. C., Larrañaga, E. \& Yubero, S. (2002). Libros, lectores y mediadores. Cuenca: UCLM.

Imbernón, F. (1989). La formación inicial y la permanente del profesorado. Dos etapas de un mismo proceso. Revista Interuniversitaria de Formación del Profesorado, 6, 487-499.

Junta de Andalucía. Decreto 93/2013, de 27 de agosto, por el que se regula la formación inicial y permanente del profesorado en la Comunidad Autónoma de Andalucía, así como el Sistema Andaluz de Formación Permanente del Profesorado.

Junta de Andalucía. Instrucciones de 24 de julio de 2013, de la Dirección General de Innovación Educativa y Formación del Profesorado sobre la Organización y funcionamiento de las bibliotecas escolares de los centros docentes públicos que imparten Educación Infantil, Educación Primaria y Educación Secundaria.

Junta de Andalucía. Ley 17/2007, de 10 de diciembre, de Educación de Andalucía.

Junta de Andalucía. Orden de 31 de julio de 2014, por la que se aprueba el III Plan Andaluz de Formación Permanente del Profesorado.

Lluch, G. (ed) \& E. Gil, J. Martín, R. Morduchowicz, G. A. Arellano y P. C. Cerrillo (2010). Las lecturas de los jóvenes. Un nuevo lector para un nuevo siglo. Barcelona: Anthropos.

Manzanares, A. \& Galván-Bovaira, M. J. (2012). La formación permanente del profesorado de Educación Infantil y Primaria a través de los Centros de Profesores. Un modelo de evaluación. Revista de educación, 359,431-455.

Marchamalo, J. (2004). El mapa del tesoro. J. Marchamalo, V. Fernández, X. A. Neira, T. Colomer y E. Pérez. Anuario sobre el libro infantil y juvenil (pp. 7-35). Madrid: SM.

Mendoza, A. (2003). Didáctica de la Lengua y la Literatura. Madrid: Prentice Hall.

Ministerio de Educación y Ciencia. Real Decreto 2112/1984, de 14 de noviembre, por el que se regula la creación y funcionamiento de los Centros de Profesores.

Moreno, A. y Sánchez, L. (2000). Treinta años de la evolución de los estudios universitarios de literatura infantil en España: 1967-1997. V. Ruzicka, C. Vázquez y L. Lorenzo (eds.) Literatura infantil y juvenil. Tendencias actuales (pp. 85-96). Vigo: Servicio de publicaciones de la Universidad de Vigo.

Willigan, D. T. (2016). Educando niños lectores. Lo que padres y maestros pueden hacer. Madrid: TEEL. 
Zeichner, K. M. (2010). Nuevas epistemologías en formación del profesorado. Repensando las conexiones entre las asignaturas del campus y las experiencias de prácticas en la formación del profesorado en la universidad. Revista Interuniversitaria de Formación del Profesorado, 68, 123-149. 